\title{
Les théories de la lumière dans une science en mouvement
}

Bernard Maitte (Bernard.Maitte@univ-lille1.fr)

Professeur émérite à l'Université de Lille 1, Sciences et technologies, P5 bis,

59655 Villeneuve d'Ascq Cedex

L'évolution des idées sur

la nature de la lumière a marqué

I'histoire de la physique,

de l'an mille à nos jours.

On y voit s'affronter des

hommes et leurs conceptions

du monde : elles dépendent

des contextes dans lesquels

ils travaillent, de l'état de la

science à une époque donnée.

Nous croiserons les Descartes,

Huygens, Newton, Euler,

Young, Fresnel, Maxwell dans

leurs efforts pour construire

une science explicative

et prédictive, exempte

de contradictions. Toutes

leurs tentatives se révéleront

a posteriori insatisfaisantes...

Mais ces échecs n'en sont pas :

ils ont permis la naissance

de nos conceptions modernes,

pertinentes entre des limites

de validité que nous savons

poser, en constante remise

en cause.

Pour en savoir plus

B. Maitte, Une histoire de la lumière, de Platon au photon, Éditions du Seuil, Paris (2015).

\section{La vision dans l'Antiquité}

Les philosophies antiques ne se sont jamais préoccupées de la nature de la lumière. Toutes les Écoles ne s'intéressent qu'à la vision, considérée comme l'action $\mathrm{du}$ semblable sur le semblable. Pour les pythagoriciens, Empédocle et Platon, nous voyons parce que l'œil émet un "feu de la vue » qui rencontre le feu extérieur. Euclide fait de son optique (300 av. J.-C.) une géométrisation du regard. Pour les atomistes, des objets émanent des enveloppes ténues qui conservent la forme de l'objet, appuient sur l'air et y rencontrent d'autres effluves provenant des humeurs internes de l'œil. Selon Aristote (384-322 av. J.-C.), la vision se distingue des autres sens en ce qu'elle permet de voir les couleurs. Pour en rendre compte, il observe les pratiques des peintres et des teinturiers : ils obtiennent le blanc par purification, les couleurs en mélangeant des pigments. Pour lui, le blanc est donc homogène, les couleurs mélanges. Il en explique la grande variété par sa philosophie : le monde est constitué de deux régions cosmiques aux propriétés bien différentes. Au centre, siège la Terre - zone de génération et de corruption -, formée de quatre éléments terre, eau, air et feu; autour se situe le Ciel - lieu de perfection -, limité par la sphère des étoiles fixes, empli du cinquième élément, l'éther. Une partie de celui-ci pénètre dans notre monde, $\mathrm{y}$ emplit les corps : on l'appelle alors le diaphane. Quand il n'y a ni regard ni lumière, celui-ci est en puissance : c'est le noir, l'absence de visibilité. Quand la lumière et le regard sont présents, le diaphane passe immédiatement en acte, donne les différentes couleurs, selon l'illumination subie et les impuretés présentes dans les corps.
Sur le plan des pratiques, l'histoire retiendra aussi Archimède (212 av. J.-C.) : il aurait incendié la flotte romaine stationnée devant Syracuse au moyen de miroirs ; Ptolémée (+ 150), qui utilise ses instruments d'astronomie pour mesurer la vision viciée par réfraction, détermine ainsi la position réelle des corps vus. Claude Galien (131-201) dissèque des yeux (+ 195); en les coupant avec un rasoir, le cristallin, dur et lenticulaire, s'échappe : Galien le place au centre de l'œil et fait se former sur lui les images.

\section{Une première théorie corpusculaire dans le monde arabe}

Lors de l'expansion musulmane $\left(7^{\mathrm{e}}\right.$ et $8^{\text {e }}$ siècles), les cavaliers préservent les cultures des pays conquis, s'approprient leurs savoirs : de nombreux textes sont traduits dans la langue culturelle, celle du Coran. Des institutions d'enseignement sont créées dans les nouvelles villes (Bagdad, le Caire, Cordoue, Damas, Ispahan, Kairouan...). Dans ce contexte, les sciences arabes (c'est-à-dire écrites en arabe, quelle que soit la confession des auteurs) sont profanes. Elles empruntent à plusieurs traditions, vont plus loin que toutes celles qui les ont précédées.

En optique, al-Kindi ( $9^{\mathrm{e}}$ siècle) étudie la lumière réfléchie des «miroirs ardents » : elle possède une réalité matérielle et se propage, dit-il, selon des rayons lumineux. Ibn-Sahl $\left(10^{\mathrm{e}}\right.$ siècle) ne s'intéresse plus à la vision mais à la lumière solaire, aux rayons réfractés. Ibn al-Haytham (965-1039) se saisit de ces prémices. Il commence par distinguer tous les effets de la lumière, les mesure au moyen d'appareillages qu'il met au point ou perfectionne (diaphragmes, mires, 


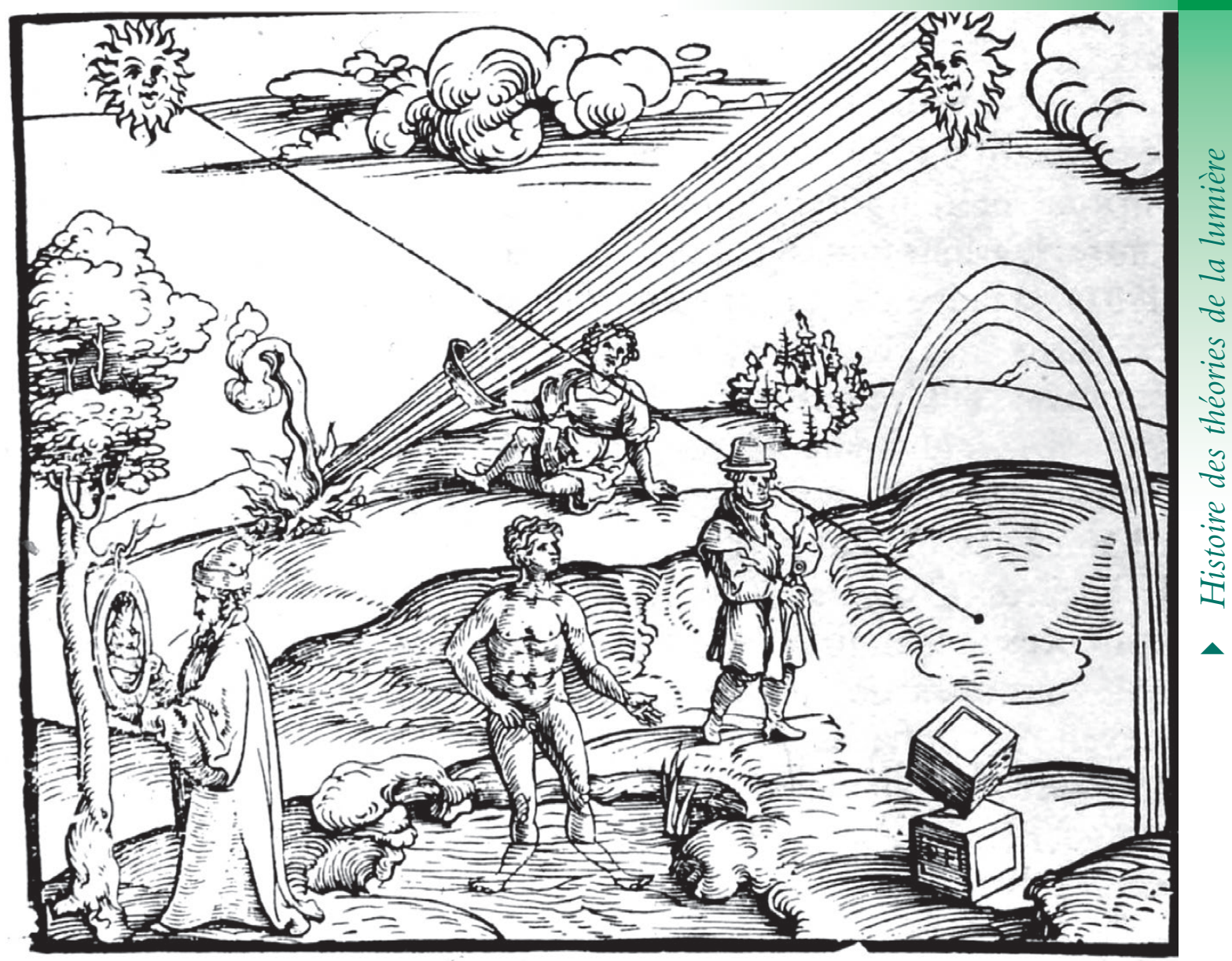

1. Les effets de la lumière selon Witelo, commentateur de Ibn al-Haytham : dispersion (arc-en-ciel), réflexion dans un miroir, réfraction, embrasement... Frontispice du livre imprimé Magie de Witelo (Nuremberg, 1535).

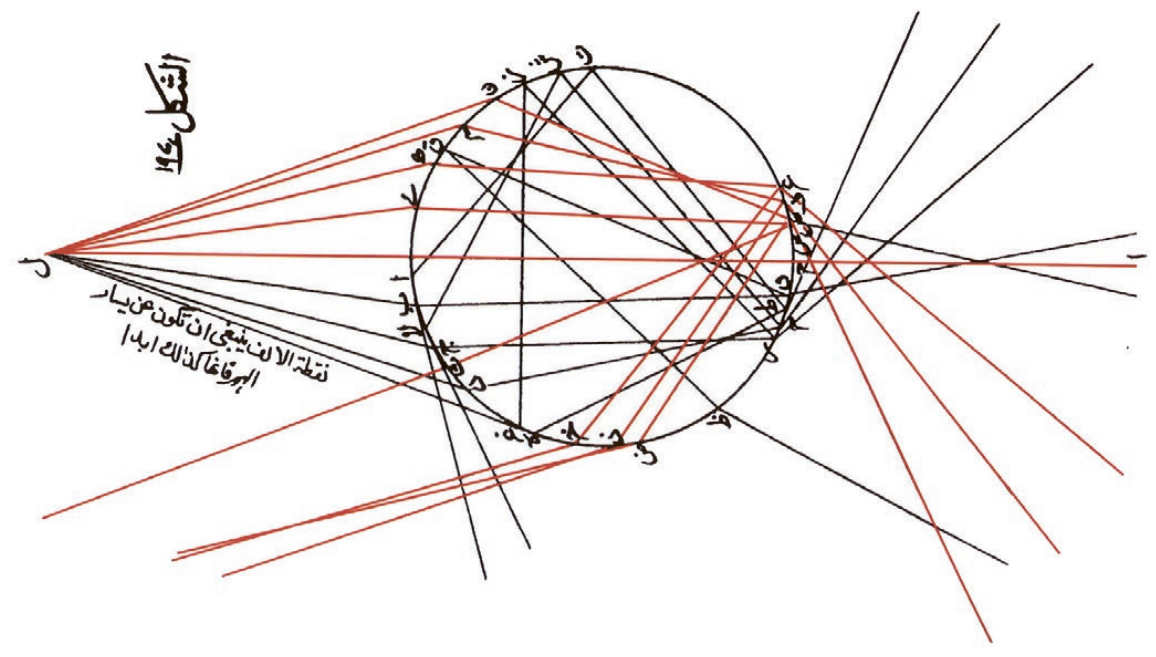

chambres noires...). Il peut ainsi caractériser propagation rectiligne, réflexion, réfraction, embrasement après des miroirs ou des sphères ardentes (figs. 1 et 2), couleurs. Voulant expliquer tous ces faits par une même cause, il remarque que la lumière fait mal, blesse, lèse : ne pourraitelle pas être constituée de petites sphères émises de la source à une très grande vitesse ? Pour vérifier, il étudie le mouvement des corps graves : leur propagation est rectiligne, ils rebondissent, peuvent traverser une toile tendue en déviant de leurs trajectoires... La mathématisation de ces comportements est la même que celle de la lumière : la "plus petite des lumières" se comporte comme le mouvement d'un corps pesant. Elle est émise par les sources, traverse l'espace, parvient à l'œil, y entre, forme l'image sur le cristallin placé au centre. Toutes ces mesures et déductions sont rapportées dans son magistral Traité d'optique, qu'il fait précéder d'un long préambule, dans lequel il expose la méthode hypothéticodéductive testée par l'expérience qu'il a suivie. C'est la méthode expérimentale que nous voyons naître sous sa plume. 
>>

\section{En pays chrétien, une théorie ondulatoire permet de concilier Foi et Raison}

Les sciences des pays d'Islam passent dans le monde latin à partir du $10^{\mathrm{e}}$ siècle, grâce à des voyageurs, aux communautés hébraïques fixées dans les deux mondes, par les contacts directs, par la reconquête de la Sicile (1063) et de Tolède (1085). Les traductions sont nombreuses à partir du $12^{\mathrm{e}}$ siècle.

Mais les orientations des clercs sont tout autres que celles des savants en pays d'Islam : au sein des monastères, ils essaient de concilier la foi et la raison par le raisonnement, non par la méthode expérimentale, qui restera inconnue pendant de longs siècles chez les Latins. La théorie d'Ibn al-Haytham est discutée, Witelo (1270) s'en fait le propagateur (fig. 1).

Thomas d'Aquin (1272) est dans une autre logique : il adapte la métaphysique d'Aristote à la révélation chrétienne, fait de la lumière une pure qualité qui représente la perfection de Dieu; elle ne peut être vulgaire, se quantifier.

À Oxford, Robert Grossetête (1253) se pose comme héritier à la fois d'Aristote, dont il retient le schéma général du monde, l'existence de l'éther, et des mécaniciens arabes quand ils géométrisent. Il veut résoudre la contradiction qui voit, dans la Genèse, Dieu séparer le premier jour la lumière des ténèbres puis créer les luminaires le quatrième jour : pour lui, la lumière est la première forme corporelle créée par Dieu, en un point. Ce point possède la propriété de se dilater et d'étendre l'espace instantanément, selon une sphère. Lorsqu'elle atteint sa limite de ténuité, cette sphère de lumière fondamentale (le lux) cesse de se dilater. La lumière matérielle (le lumen) se rétracte alors, revient au point initial, se dilate à nouveau et ainsi de suite. À la quatrième rétractation se forment les planètes, puis la sphère terrestre. Cette métaphysique de la lumière est mise par Grossetête au service des sciences de la nature : comme le monde est plein, il identifie le lumen à des battements analogues aux sons qui se propagent dans l'éther ; il lui applique une géométrisation utilisant sphères, droites, mesures, pour expliquer réflexions et réfractions. Voici une pre- mière conception " ondulatoire " de la lumière et lancé un débat entre trois conceptions de la lumière.

Sur le plan des techniques, des artisans inventent, dés le $13^{\mathrm{e}}$ siècle, les «lentilles de verre ", appelées ainsi par analogie avec le légume. Les clercs les utilisent pour améliorer la vue basse. Aucun ne les étudie : selon Aristote, la vue est fallacieuse. Étudier un artifice placé devant les yeux est "indigne de philosophie».

\section{Les perspectivistes et la naissance de la science moderne}

À Florence, au Quattrocento, les peintres perspectivistes font apparaître dans leurs tableaux un espace homogène ne distinguant plus régions céleste et terrestre (fig. 3). Ficin développe un culte solaire, tout en considérant en optique les rayons visuels partant de l'œil.

À leur suite, Copernic (1543), revenant d'un long séjour en Italie, propose un Univers unifié où le Soleil occupe la place centrale. Cette proposition n'entraîne guère, initialement, l'opposition des milieux protestants et catholiques, à une époque ou Réforme et Contre-Réforme reviennent au sens littéral des Écritures. Mais les insuffisances du système héliocentrique sont nombreuses : comment expliquer la chute d'un corps sur une Terre en mouvement?

C'est sur ces difficultés que Galilée travaille tout d'abord (1590). Il se fait le propagandiste de la méthode expérimentale et va chercher chez les artisans les méthodes de mesures. Apprenant l'existence de la lunette, il montre (1622) au Sénat de Venise qu'elle permet de voir plus gros les objets éloignés, la tourne vers le ciel, effectue une multitude d'observations (fig. 4) qui confirment le monde de Copernic. Pour lui, la lumière, dont il propose une manière de mesurer la vitesse, est la messagère des étoiles. Il s'adresse au peuple des villes, lutte contre les aristotéliciens, sépare la foi de la raison, décrit un monde unifié, " écrit en langage mathématique " connaissable grâce à notre seule raison (1632). Il pose ainsi les bases de la science moderne.

Kepler (1604) fait définitivement de la lumière un objet physique, détermine le chemin optique des rayons lumineux au travers de l'œil, fait se former l'image

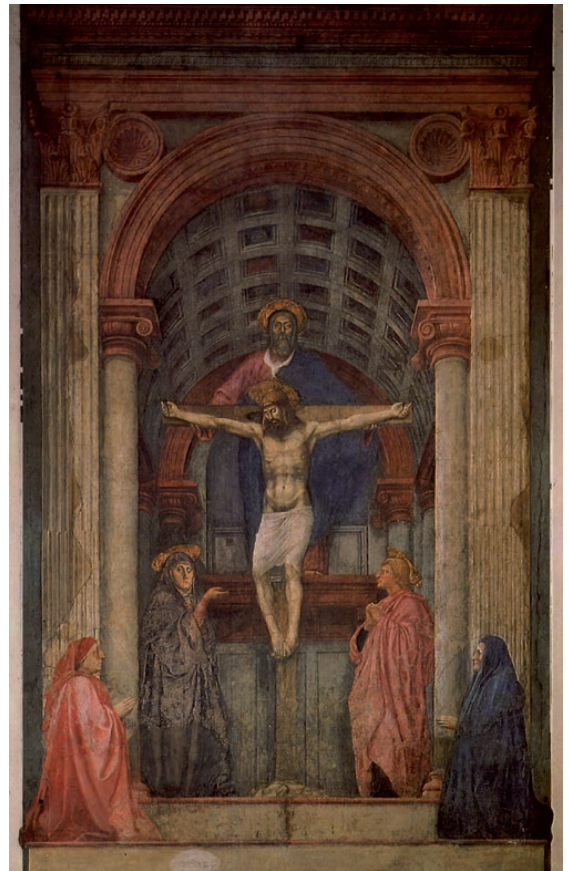

3. La Trinité de Masaccio (1426) dans l'église Santa Maria Novella à Florence. Le peintre met Dieu dans l'espace des hommes, et le coiffe d'une architecture à la Brunellaschi. L'espace est isotrope.

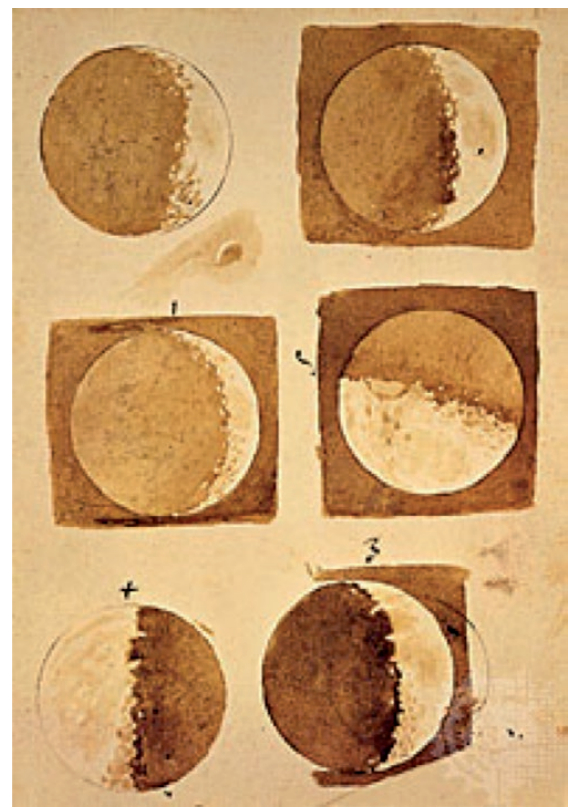

4. La Lune, selon Galilée (1610). Elle est couverte de montagnes, comme la Terre. 
inversée sur la rétine, distingue nettement dans la vision les aspects physiques, physiologiques et cérébraux. Après que Galilée a levé l'ostracisme pesant sur les lentilles de verre, il fait, dans sa Dioptrique (1611) la théorie des instruments optiques. Mystique, la lumière est pour lui une représentation de la Trinité : le Soleil correspond à Dieu le Père, l'œil au Fils, l'espace intermédiaire au Saint-Esprit.

Avec Galilée et Kepler, c'est un basculement de la manière de démontrer qui se produit : notre science moderne est celle du compter, du mesurer, des applications.

\section{La théorie ondulatoire de Huygens}

Après la condamnation de Galilée, le prudent Descartes renonce à publier Le Monde ou Traité de la lumière, un ouvrage copernicien. Il aborde les problèmes d'optique dans son Discours de la Méthode (1647) : l’Univers est plein, empli de trois éléments : la terre, dont sont faits tous les corps et les planètes ; l'air subtil, ou éther, analogue à de petites sphères en contact animées de mouvements circulaires, uniformes et éternels. Cet éther s'insinue partout, emplit l'espace, se répartit en tourbillons, cause par entraînement le mouvement des planètes (fig. 5). Le troisième élément, le $f e u$, est encore plus petit : il passe entre les sphères de l'éther, se concentre aux coins des tourbillons. L'Univers est infini, toutes les étoiles sont des soleils, occupent le centre des tourbillons; leur lumière nous provient par une pression instantanée s'exerçant dans l'éther (Descartes croit pouvoir l'affirmer en interprétant les observations d'éclipses de Lune.). Nous voyons comme un aveugle sent un obstacle au moyen de son bâton, les yeux transmettent la sensation au cerveau, l'âme interprète. En ce qui concerne les effets lumineux, Descartes les explique au moyen de modèles mécaniques de sphères qui se propagent, contredisant donc le contact immédiat. Il publie le premier la loi de la réfraction, qu'il emprunte à Snell, mort avant de la publier.

Continuateur irrévérencieux de Descartes, Christiaan Huygens veut lever les contradictions qu'il trouve dans les travaux de son maitre. Pour cela, il utilise la méthode expérimentale de Galilée. Postulant un monde plein, empli

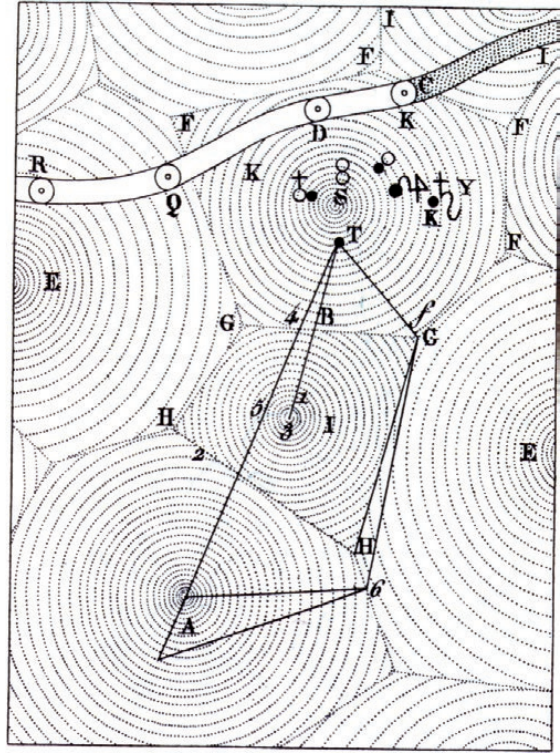

5. Le monde de Descartes est empli de tourbillons, qui entraînent les planètes. (R. Descartes, Le Monde ou Traité de la lumière, 1664.)

d'éther qui s'insinue partout, il remarque (1690) que les sources lumineuses sont les lieux d'une agitation intense : les chocs engendrés par ces mouvements heurtent les particules d'éther et se propagent dans toutes les directions. À l'aide de la géométrie, Huygens parvient à rendre compte de la propagation rectiligne, de la réflexion, de la réfraction (en déduisant que la lumière va moins vite dans l'eau que dans l'air) par une élégante théorie ondulatoire. Les cartésiens peuvent proposer que l'Univers est plein : c'est une immense horloge créée par Dieu, qui se repose le septième jour et n'intervient plus dans la physique. Tout dans le monde peut se décrire en termes de géométrie, de mécanique, de chocs et de mouvements. Ainsi, la lumière est une onde qui se propage. Faut-il donc rompre avec la théorie corpusculaire d'Ibn al-Haytham ?

\section{La théorie corpusculaire dans la gravitation universelle de Newton}

"En ce qui concerne la physique, Monsieur Descartes n'a rien écrit de bon. "Celui qui se permet cette appréciation péremptoire est un jeune homme, Isaac Newton. En 1666, à 23 ans, à la suite de Boyle, il se montre atomiste. Il s'intéresse aux couleurs, postule une théorie corpusculaire, reproduit l'expérience, classique, de dispersion des couleurs par un prisme, mais innove alors : il se place dans l'obscurité. Sur le mur situé derrière le prisme, il s'émerveille à "contempler les couleurs vives et intenses ainsi produites ": rouge à une extrémité, puis jaune, verte, bleue, violette pour les rayons les plus déviés. Il appelle spectre coloré cette image qui traverse la pièce comme un fantôme. Guidé par sa théorie, il isole une des couleurs dispersées au moyen d'un diaphragme et fait tomber

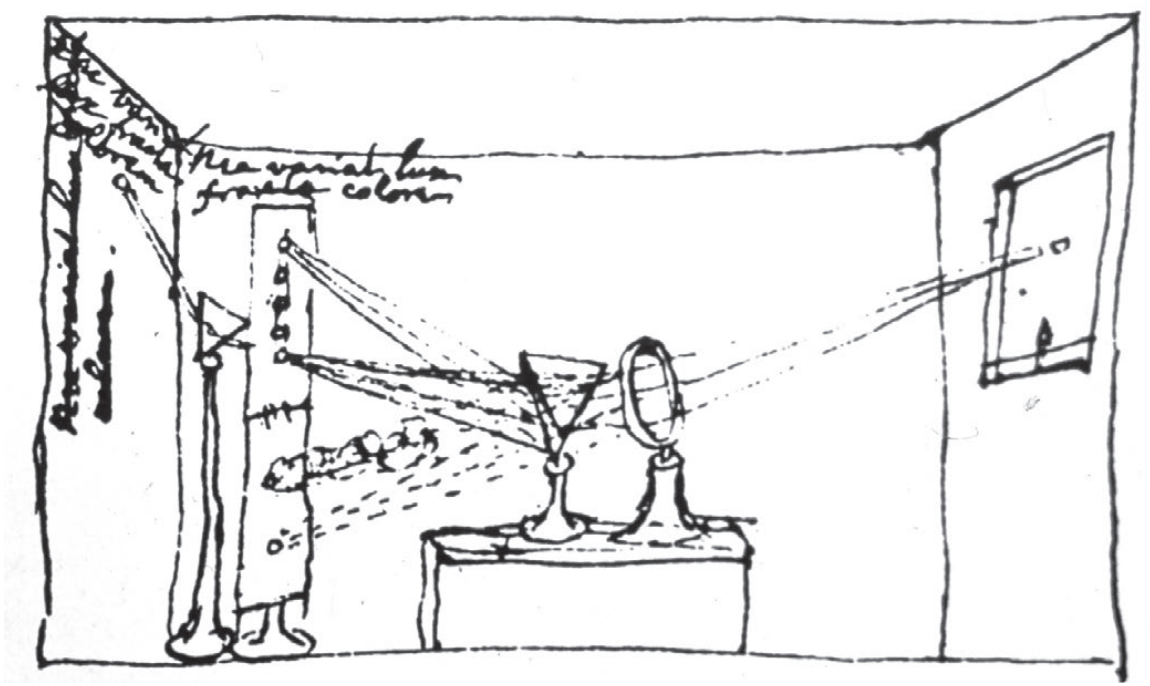

6. La dispersion par un prisme. Dessin autographe d'Isaac Newton (vers 1666). De droite à gauche : on distingue l'ouverture par où passe la lumière solaire, le premier prisme, la planche percée de trous afin de sélectionner un faisceau émergeant d'une seule couleur, puis le second prisme et l'absence de dispersion observée sur le mur opposé à l'ouverture. 


\section{>>}

un rayon monochromatique sur un second prisme, qui ne le disperse plus (fig. 6). Newton note qu'à chaque couleur correspond un degré de réfrangibilité. Multipliant les observations, il a la grande surprise de constater que le mélange des couleurs donne le blanc. Ceci renverse toutes les conceptions admises depuis Aristote : les couleurs peuvent être pures, le blanc est toujours composé ! Pour Newton, la lumière est formée de petites masses déviées par l'action de forces. Ces déductions logiques sont tout à fait différentes de celles effectuées par les cartésiens. Elles sont refusées par la Royal Society, qui admet pourtant Newton en son sein pour son télescope à réflexion, qu'il a inventé parce qu'il croyait pouvoir affirmer que toute lentille forme obligatoirement des images irisées par la dispersion.

Écœuré, Newton « renonce à la philosophie " (1672). Il y revient avec la publication de ses Principia (1687) : sa Loi de la Gravitation Universelle y décrit tous les effets physiques observés, de l'infiniment grand - les astres - à l'infiniment petit - la lumière. Celle-ci est formée de petites masses lancées par la source; elles se propagent à une vitesse fantastique, comme vient de le montrer Römer grâce à l'observation des satellites de Jupiter. Lorsqu'ils arrivent, par exemple sur un prisme, les corpuscules sont déviés. Ils se rapprochent de la normale à la surface de séparation, sont donc accélérés par une force : la lumière va plus vite dans le verre que dans l'air, conclue Newton.

En 1702, il publie son Optique, somme de toutes ses recherches en optique. Il y étudie, en particulier, les teintes que présentent les bulles de savon, note leur périodicité, leur relation avec l'épaisseur traversée. Il ne parvient à en rendre compte que par une "théorie des accès", qui réintroduit l'éther et les vibrations d'ondes en son sein. Mais il ne remet pas en cause son système du monde pour une bulle de savon ! Il préfere écrire qu' " un homme qui voyait mieux que moi " a pu tracer dans le spectre les limites de sept couleurs. Dieu a donc fait une création parfaite : elle comprend sept planètes, sept notes de musique, sept couleurs. La physique de Newton conduit à une théogonie.

\section{La lumière des Lumières}

Le $18^{\mathrm{e}}$ siècle ne va pas accepter facilement les positions de Newton. Un grand débat a lieu, en particulier entre les aristotéliciens, dont les positions s'effritent, les cartésiens, les newtoniens. Au sujet du mouvement des planètes, ceuxci récusent Descartes : si les planètes étaient entrainées par l'éther, il y aurait contacts, frottements, dissipation de chaleur, perte de mouvement : l'horloge de Descartes devrait s'arrêter. Le monde ne peut donc être plein : il est vide ! Les cartésiens ne peuvent admettre ces conclusions : la gravité, selon Newton, doit s'exercer entre la Terre, la Lune et le Soleil, dans le vide; elle ne peut être de nature mécanique. Elle est " action constante de Dieu sur le monde ", affirment les newtoniens, pour qui le divin créateur intervient sans cesse sur sa création pour compenser le dépérissement naturel des forces mécaniques qui s'y exercent...

Les scientifiques du continent essaient donc de prendre en défaut la "philosophie anglaise "; leurs tentatives échouent. Mieux, grâce à la gravitation universelle on parvient à déterminer la forme du globe, à expliquer la précession des équinoxes, à prévoir le retour de la comète de Halley : la physique de Newton s'impose par ses succès. Laplace développe (1796) un système du monde newtonien dans lequel la gravité est une propriété inhérente à la matière : Dieu est chassé de la physique. La théorie corpusculaire de la lumière s'impose, malgré la découverte de la possibilité de fabriquer des lentilles achromatiques, ce qui est contraire à l'optique de Newton, et malgré l'opposition d'Euler qui, dans sa Nova Theoria (1746), développe, par analogie avec le son et les cordes vibrantes, une théorie ondulatoire exprimée par l'analyse : les couleurs seraient dues à des vibrations de même fréquence...

\section{Le renouveau des théories ondulatoires}

À l'heure même où la physique newtonienne s'impose, en Angleterre, Thomas Young, un médecin, se pose le problème mécanique suivant : dans l'eau, la rencontre d'ondes produit soit une augmentation soit une diminution des mouvements de la surface. Qu'en est-il de la lumière ? Pour répondre, il fait passer un faisceau par deux trous, les rayons issus de ceux-ci se superposent dans une zone de l'espace. Il observe alors (1802) des franges alternativement sombres et claires : "de la lumière ajoutée à de la lumière donne de l'obscurité ", ce qui n'est pas explicable par la théorie corpusculaire, mais peut l'être par la théorie des ondes. Ce résultat et son interprétation sont violemment rejetés par les savants anglais.

En 1809, voulant interpréter une expérience de Huygens redécouverte, la biréfringence de la calcite, Malus découvre que la lumière perd ses propriétés de symétrie par une simple réflexion, par biréfringence ; il explique ces faits en complétant la théorie corpusculaire : les corpuscules lumineux auraient des formes et s'aligneraient lors des phénomènes cités. C'est ce qu'il appelle polarisation de la lumière. Une victoire de la théorie corpusculaire.

En 1814, en France, Arago reçoit un mémoire dont l'auteur, Augustin Fresnel, a étudié la physique de Descartes. La théorie newtonienne de la lumière, apprise à Polytechnique, ne l'a pas convaincu : il écrit des "rêveries " destinées à renverser la théorie corpusculaire... Bientôt il fait tomber sur un cheveu un mince pinceau lumineux et regarde l'ombre... celle-ci est bordée de franges, dont l'aspect ressemble aux ondes de l'eau. Arago l'encourage : Fresnel précise ses observations, écrit les équations de propagation des ondes, prévoit les positions et les intensités des franges (fig. 7), vérifie par l'expérience. La concordance des prévisions et des observations est excellente. L'Académie des sciences couronne son mémoire sur la «diffraction de la lumière" (1818), mais la majorité de ses membres se refuse à remettre en cause Newton pour l'ombre d'un cheveu, ceci d'autant plus que Fresnel, s'il explique presque tous les effets lumineux par sa théorie ondulatoire, ne sait rendre compte de la polarisation.

Après bien des efforts, Fresnel parvient à trouver une solution : la polarisation peut être justifiée en considérant que les ondes lumineuses ne sont pas associées à des vibrations longitudinales, comme celles du son, mais à des vibrations transversales (1821). Arago refuse de le suivre dans ces " acrobaties ". Pourquoi cette réticence ? Dans les milieux fluides 


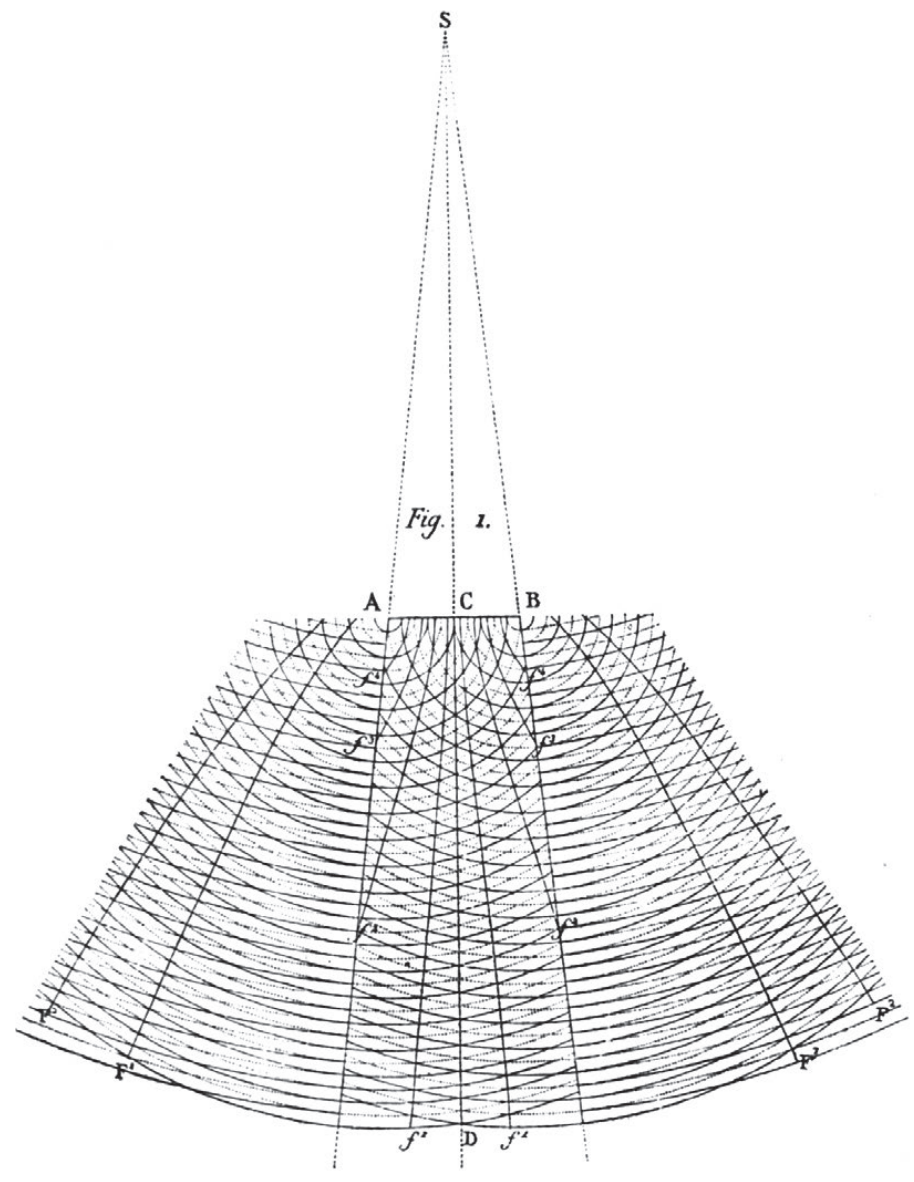

7. La diffraction selon A. Fresnel. S est la source lumineuse, A et B les extrémités du corps qui porte ombre. Les cercles représentent les nouds et ventres des ondes issues de A et B. Leurs intersections définissent les franges d'interférences (" Premier mémoire sur la diffraction de la lumière " (1815), dans A.-J. Fresnel, CEuvres complètes, vol. 1, Imprimerie impériale, Paris (1868)).

(eau, air) les vibrations sont longitudinales. Mécaniquement parlant, les vibrations transversales ne peuvent se propager que dans des milieux visqueux. La théorie ondulatoire implique donc que la lumière nous parvient des étoiles, que ses ondes sont transmises dans un éther emplissant l'espace, que cet éther est analogue à de la gelée visqueuse, mais que la Terre circule librement dans

l'espace au milieu de cette gelée... C'est que l'éther passe librement au travers de notre globe ose Fresnel! Arago repense à la mesure de la vitesse de la lumière dans l'eau : plus grande que dans l'air pour les " corpuscularistes ", plus lente pour les " ondulationistes ". Il suggère à Foucault de faire l'expérience. En 1849, celui-ci détermine qu'elle est moins grande que dans l'air, comme le prédit la théorie ondulatoire. Cette expérience permet de rejeter définitivement la théorie corpusculaire. Il va falloir se pencher sur les bien curieuses propriétés de l'éther!

\section{L'éther électromagnétique}

Revenons un peu en arrière. Au début du $19^{\mathrm{e}}$ siècle, le système de LaplaceNewton présente d'autres difficultés qu'en optique : pour expliquer la chaleur, l'électricité et le magnétisme, les newtoniens font appel à des fluides impondéraux. Toute cette approche est rejetée par la "Naturphilosophie ": la matière ne peut être constituée d'atomes, est divisible à l'infini, les phénomènes de la physique peuvent s'expliquer par des conflits entre des forces antagonistes s'exerçant dans un espace continu.

Cherchant à mettre en évidence de tels conflits, le Danois Oersted (1819) observe qu'un courant électrique fait dévier l'aiguille d'une boussole perpendiculairement à la droite qui joint les corps en interaction (le fil électrique et la boussole), alors que la physique newtonienne ne fait intervenir que des forces dans la direction de cette droite. Arago introduit ces expériences en France, Ampère (1820) les mathématise et les étend. Michael Faraday (1821) prolonge cette démarche. Manquant de formation mathématique, il regarde les figures que dessine la limaille de fer autour des courants (fig. 8), constate que les petites aiguilles se disposent en "lignes de forces ", dont l'aspect lui rappelle les labours vus d'un ballon au-dessus de la campagne : par analogie, il appelle " champ de forces " l'action progressive du milieu séparant le conducteur de la limaille. Pour lui, cet espace intermédiaire serait analogue à un poulpe muni de nombreux tentacules. Sous l'effet d'une variation de courant, ses bras se déplaceraient et entraîneraient la limaille... Ầ une époque où la physique est fortement mathématisée, les déductions de Faraday paraissent naïves.

Elles attirent cependant l'attention de J-C. Maxwell, qui en retient plusieurs aspects : le rôle du milieu intermédiaire, la notion de champ qui se propage, l'interaction entre électricité et magnétisme. Le seul tort de Faraday, pense Maxwell, est d'avoir expliqué la structure de l'espace d'une manière non mathématisable. Maxwell s'évertue donc à

8. Les champs de force de Faraday. 
$>>$

trouver "une analogie physique ", dont l'action serait la même que celle qui oriente la limaille ; il parvient à imaginer un tel dispositif, formé de roues et de pignons (1862) (fig. 9). Il en donne la description mathématique, réduit les roues à de simples points, obtient des formules de propagation continues du champ. Elles rendent compte de tous les effets observés autour des aimants, des courants : il peut rejeter son analogie mécanique devenue inutile et ne garder que les formules. Une nouvelle branche de la physique vient de naître : l'électromagnétisme. Ses formules lui permettent de calculer la vitesse de la propagation du champ électromagnétique : $300000 \mathrm{~km} / \mathrm{s}$. Que de coinncidences avec la lumière : même vitesse, un éther, des ondes, des vibrations transversales... Pourquoi remplir l'espace d'un nouveau milieu toutes les fois que l'on doit expliquer un nouveau phénomène ? En 1873, il franchit le pas et postule qu'éther luminifere et éther électromagnétique ne sont qu'un, que la lumière est une onde électromagnétique... Plus besoin de chercher à définir la rigidité de l'éther, à en faire de la gelée visqueuse : il devient simple support. Maxwell vient d'unifier lumière, électricité, magnétisme... et de rompre avec la volonté de décrire le monde en termes de mécanique. La majorité des physiciens de son temps qualifie Maxwell de "cinglé ».

En 1885, Heinrich Hertz, veut vérifier les équations de Maxwell : il fait des étincelles entre les bornes d'un crépiteur, produit ainsi des ondes électromagnétiques, décrit leur propagation, prouve leur réflexion, leur réfraction, leur transversalité, mesure leur longueur d'onde (environ un mètre) et leur vitesse de propagation : $300000 \mathrm{~km} / \mathrm{sec}$. Dix ans plus tard, Roëntgen découvre les rayons $\mathrm{X}$, qui seront identifiés comme des ondes électromagnétiques à très courte longueur d'onde. Des rayons $\mathrm{X}$ aux ondes hertziennes, les ondes électromagnétiques montrent des variations continues de longueur d'onde dont les effets perceptibles sont différents et peuvent être mis en évidence par différents types de récepteurs. Le récepteur qu'est l'œil n'est sensible qu'à une bande très étroite de longueurs d'ondes allant de 0,4 à 0,75 micron : c'est ce que l'on appelle «lumière ». À la même époque, les phénomènes thermodyna-

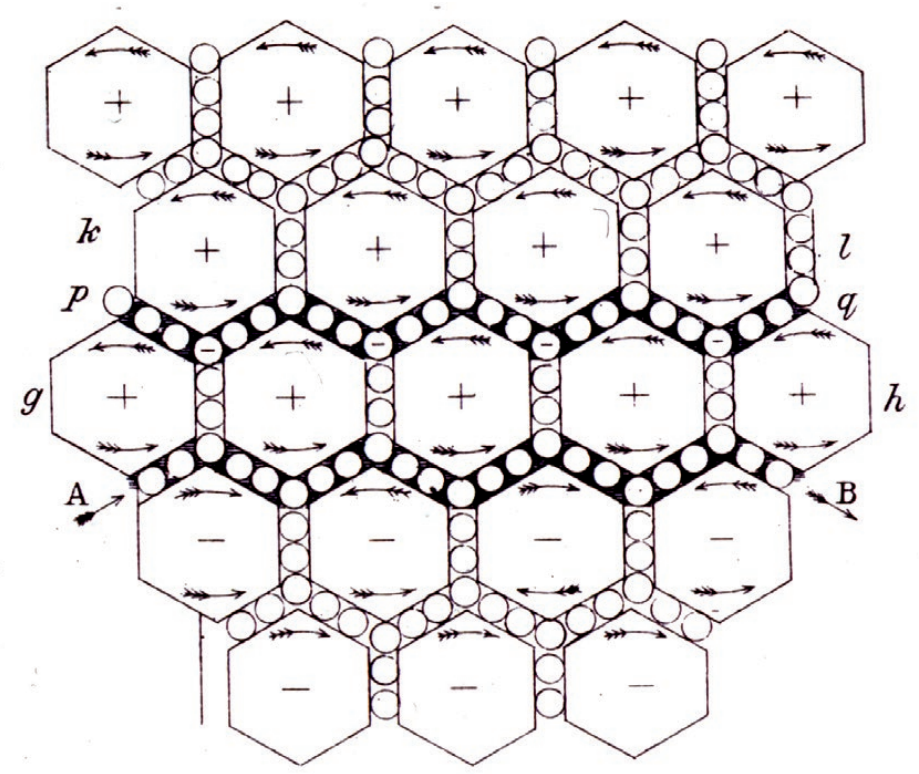

9. Les roues et les pignons de Maxwell produisent le même effet que le champ électromagnétique (J.C. Maxwell, "On Physical Lines of Force", Phil. Mag. (1861)).

miques sont, eux aussi, expliqués par des équations continues : dans la seconde moitié du $19^{\mathrm{e}}$ siècle, rares sont les physiciens qui osent se montrer atomistes. Sommes-nous parvenus à la fin de l'histoire de la lumière ? Les physiciens le croient à l'aube du $20^{\mathrm{e}}$ siècle. Traduisant l'opinion générale, Lord Kelvin affirme : "la physique est définitivement constituée dans ses concepts fondamentaux... Il y a bien deux petits problèmes : celui $d u$ résultat négatif de l'expérience de Michelson et celui du corps noir, mais ils seront rapidement résolus et n'altèrent en rien notre confiance. " Premier problème : l'expérience de Michelson. Si l'éther emplit le monde, le mouvement de la Terre doit amener des perturbations. Dix ans d'efforts, des mesures extraordinairement précises ne permettent pas de caractériser le vent d'éther, mais montrent que la vitesse de la lumière mesurée à partir d'une source en mouvement (la Terre) est toujours constante et ne suit pas la loi d'addition des vitesses. Second problème : celui du « corps noir». Le corps noir est un objet théorique d'étude. Pour comprendre le problème qu'il pose, il suffit d'imaginer une barre de fer que l'on chauffe : au fur et à mesure que la température s'élève, elle devient rouge, puis orange, jaune, blanche, bleue avant de reprendre sa couleur initiale... À une certaine température, l'énergie rayonnée par la barre peut se représenter, en fonction des fréquences par une courbe en cloche. Si on calcule cette énergie à partir des données de la théorie électromagnétique, on trouve qu'elle devrait tendre vers l'infini en allant vers l'ultraviolet... C'est ce que le facétieux Ehrenfest appelle la « catastrophe ultraviolette ", catastrophe pour la théorie, sur laquelle il faut se pencher.

\section{Vers une nouvelle physique}

La résolution des deux petits problèmes va totalement bouleverser la physique : point n'est besoin de m'y attarder longuement.

En 1905, Einstein résout la première contradiction grâce à sa " théorie de la relativité ". Il rejette un éther devenu inutile, propose de considérer la vitesse de la lumière dans le vide comme une vitesse limite, montre que les mesures de l'espace et du temps varient avec la vitesse du système de coordonnées dans lequel on les repère, qu'ils sont liés dans un même "continuum espace-temps ". En 1915, sa théorie de la relativité générale fait de la gravitation la courbure de l'espace-temps et donne à la lumière une trajectoire qui suit la géodésique de cet espace.

Pour résoudre le problème du corps noir, Planck ruse. En 1900, il part des résultats expérimentaux et parvient à les retrouver mathématiquement en utilisant un artifice de calcul, sans considération théorique sous-jacente. En 1902, Einstein lui fait remarquer que ses calculs 
supposent que l'énergie des ondes électromagnétiques émises par le corps noir ne varie pas de façon continue, mais par valeurs précises, multiples entiers d'une énergie minimale. En 1905, Einstein rend compte d'une autre manière du corps noir, en considérant la partie de la courbe où les mesures et les prévisions coïncident : il est amené alors à considérer que l'énergie lumineuse a une distribution par quanta. Donner à la lumière une structure corpusculaire lui permet de rendre compte de l'effet photoélectrique, ce qui est loin de convaincre le monde scientifique! En 1913, Niels Bohr parvient à expliquer les spectres lumineux d'absorption et d'émission en suggérant que la matière est formée d'atomes constitués d'un noyau et d'électrons ne pouvant occuper que certains niveaux d'énergie, multiples entiers du quantum de Planck. La "maladie quantique " gagne la matière, alors même que les prédicats d'Einstein pour l'explication de l'effet photoélectrique sont vérifiés (1916) : les corpuscules de lumière sont baptisés du nom de photons.

La résolution des « deux petites contradictions " amène les physiciens à considérer que les deux objets que la physique " classique " avait distingué de tout temps, les ondes et les corpuscules, se réduisent à un seul (que l'on pourrait appeler quanton) possédant certaines propriétés des ondes (mais pas toutes), d'autres des particules (mais pas toutes). $\mathrm{Au}$ dualisme de la physique classique succède le monisme quantique.

Ainsi va la science, par une dialectique $\mathrm{du}$ doute et de la certitude : nous sommes assurés que les connaissances actuelles sont perfectibles; elles reposent donc à la fois sur des acquis stables et sur des fragilités. Et, cette histoire de la lumière le montre, la résolution de celles-ci fera toujours pousser plus loin les pertinences entre des limites de validité que nous savons poser. Ce seront les fruits de tâtonnements, d'hésitations, d'erreurs, d'apports extérieurs, de contextes dans lesquels se déroule la recherche scientifique : toutes les voies empruntées, avec plus ou moins de succès, dans l'histoire des théories de la lumière que nous venons de balayer rapidement ont procédé ainsi... et c'est parfois les voies de traverse qu'empruntent les avancées théoriques. 